\title{
Partie II : Sélection de textes sur le Tiers Monde
}

\section{(2) OpenEdition \\ 12 Journals}

Édition électronique

URL : http://journals.openedition.org/aspd/1052

DOI : $10.4000 /$ aspd.1052

ISSN : 1663-9669

\section{Éditeur}

Institut de hautes études internationales et du développement

\section{Édition imprimée}

Date de publication : 1 janvier 1994

Pagination : $318-325$

ISSN : 1660-5934

\section{Référence électronique}

«Partie II : Sélection de textes sur le Tiers Monde », Annuaire suisse de politique de développement [En ligne], 13 | 1994, mis en ligne le 19 décembre 2012, consulté le 08 septembre 2020. URL : http:// journals.openedition.org/aspd/1052 ; DOI : https://doi.org/10.4000/aspd.1052

Ce document a été généré automatiquement le 8 septembre 2020.

(C) The Graduate Institute | Geneva 


\section{Partie II : Sélection de textes sur le Tiers Monde}

\section{(parus en Suisse ou publiés par des Suisses concernant d'autres sujets que les relations Suisse- Tiers Monde)}

1 135. Actes de la Conférence de Lausanne '93 sur le Kurdistan. - [S.l.] : [s.n.], [1993]. $11 \mathrm{p}$.

2 136.Africa's agricultural development in the 1990s : can it be sustained ?: proceedings of a workshoop, 14-18 May 1991 / organized by CASIN, Centre for Applied Studies in International Negotiations; SAA, Sasakawa Africa Association; Global 2000; ed.: Nathan C. Russel and Christopher Ft. Dowswell. - Geneva : Centre for Applied Studies in International Negotiations [etc.], 1992. - VI, 208 p.

3 137. AKKARI, Abdeljali I - Paysans et techniciens agricoles dans le nord de la Tunisie : vers une nouvelle approche pédagogique. - Genève : Université de Genève, 1992. - 297 p., Thèse Fac. psychologie et sciences de l'éducation.

4 138. Amnesty International, Section suisse, Berne. - Peine de mort: un dossier pédagogique d'Amnesty International / [collab. pour la version française: Béatrice Leresche, Marie-Madeleine Viredaz]; [trad. Geneviève Kissling]. - Berne : Amnesty International, Section suisse, 1989. - 2 vol., Dossier pédagogique.

5 139.ARNOLD, Peter. - Strategien der Ressourcennutzung bei den Baute und Betsileo : soziologische und anthropologische Überlegungen am Beispiel zweier afrikanischer Bauerngesellschaften. - Bern : Universität Bern, Geographisches Institut, Gruppe für Entwicklung und Umwelt, 1992. - IV, 23 p. - (Berichte zr Entwicklung und Umwelt ; Nr. 5).

6 140.AUROI, Claude. - The state of the art in development studies and paradigmatic prospects. - Geneva : Institute of Development Studies, 1993. - 24 p. 
7 141. Un autre regard sur le développement en Afrique: [un] débat / avec Axelle Kabou ; animé par Christian Coméliau ; textes présentés par Paul Géradin. - Bruxelles : Institut catholique des hautes études commerciales-Tiers-Monde ; C.N.C.D., 1992. -172 p.

8 142.BACH-BLOCHER, Theres; WINKLER, Rahel ; ERZINGER, Matthias. - Türkei 1989 : Repression und Widerstand: Berichte von Reisen im Januar und Mai 1989. Zürich : Zürcher Freiplatzaktion, 1989. - 24 p.

9 143. BALDUZZI, Franco. - Soil mechanics of tropical soil : work on erosion, protection and construction of perrenial roads in West Africa and Brazil / ed. by Prof. H.J. Lang. Zürich : ETH, Institut für Geotechnik, 1993. - (Mitteilungen des Instituts für Grundbau und Bodenmechanik ; Nr. 142).

10 144.BECK-KARRER, Charlotte; SCHÄDELI, Sibyl. - Frauenbeschneidung in Afrika. Frauenbeschneidung im Islam. - Bern : Universität Bern, Institut für Ethnologie, 1992. 68 p. - (Arbeitsblätter/Institut für Ethnologie ; Nr. 5).

11 145. BELAY TEGENE. - Erosion : its effects on properties and productivity of Eutric Nitosols in Gununo area, southern Ethiopia, and some techniques of its control. Berne : University, Institute of Geography : Geographica Bernensia, 1992. - XIV, 173 p. (Geographica Bernesia. A, African studies series ; 9).

12 146. BOENI, Barbara. - Femmes et transfert de technologie : introduction d'une presse à l'huile de palme dans un village Toura (Côte d'Ivoire). -Zürich : ETHZ, 1993. - XIII, 140 p., Thèse se. techn. ETHZ.

13 147. BOKATOLA, Isse Omanga. - L' Organisation des Nations Unies et la protection des minorités. - Genève : Université de Genève, 1992. - XII, 291 p., Thèse droit, 1991.

14 148. BOLAY, Jean-Claude; CUNHA, Antonio; WAAS, Eveline. - Habitat populaire et pauvreté urbaine en Amérique latine: vers une nouvelle politique du logement en Bolivie / avec la collab. de Mabel Cruz, Rosario Sanchez, Alberto Nogales. -Lausanne : Institut de recherche sur l'environnement construit, Dépt d'architecture, EPF, 1993. XVI, 158, [69] p. - (Rapports de recherche/IREC ; $n^{\circ} 103$ ).

15 149. BOLLINGER, Armin. - Indios, Indios, Indios : gesammelte Schriften zum Wirken der Indios, zur Verfolgung der Indianer und zum Problem der indianischen Identität. Chur : Rüegger, 1992. - 152 p.

16 150. BUJO, Bénézet - Die ethische Dimension der Gemeinschaft: das afrikanische Modell im Nord-Süd-Dialog. - Freiburg Schweiz [etc.] : Universitätsverlag, 1993. -222 p. - (Studien zur theologischen Ethik ; 49).

17 151. CARTON, Michel ; MAURER, Jean-Luc. - Industrialisation and human resources development in Indonesia: what future for technical vocational education and training ?: 7th General Conference, Berlin, September 15-18, 1993 [European Association of Development Research and Training Institutes]. - Geneva: Institut universitaire d'études du développement, 1993. - 27, 4 p.

18 152. Centre for our common future, Geneva. - Erdgipfel 1992: Agenda für eine nachhaltige Entwicklung : eine allgemein verständliche Fassung der Agenda 21 und der anderen Abkommen von Rio $=$ The Earth Summit's Agenda for change $=$ Sommet de la terre 1992 : un programme d'action. - Geneva : Centre for our common future, 1993.-70 p. 
19 153. Comité international de la Croix Rouge (CICR), Genève. - Golfe 1990-1991: de la crise au conflit: l'action humanitaire du CICR / [réd. : Paul-Félix Michel]. - Genève : Comité international de la Croix Rouge, [1991]. - 47 p.

20 154. DALl'Aglio, Andrea. - Kunturpa llantu: l'ombre du condor: essai sur la représentation sociale de l'innovation dans quelques groupes kechwas du Pérou et de Bolivie. - Genève : Institut universitaire d'études du développement, 1992. - 350 p., Mémoire de recherche; no. 75.

21 155. DECURTINS, Silvio. - Hydrogeographical investigations in the Mount Kenya subcatchment of the Ewaso Ng'iro River. - Berne: University, Department of Geography: Geographica Bernensia, 1992. - XVIII, 151 p. - (Geographica Bernensia. A, African studies series ; 10).

156. DENTAN, Anne; B OL A Y, Jean-Claude. - De l'urgence au développement: reconstruction après catastrophes naturelles en Amérique latine : résumé et synthèse / avec la collab. de Alain Garnier. - Lausanne : Institut de recherche sur l'environnement construit (IREC) ; E.P.F.L., 1993. - 78 p. - (Rapport de recherche ; no. 104).

157. DUCOMMUN, Gil. - Une semaine chez les agriculteurs "indígenas" de la Sierra équatorienne : approche tragmentaire de l'agriculture andine (altitude 2900 à $3700 \mathrm{~m}$, Canton Salcedo, août 1992). - Zollikofen : Ecole suisse d'ingénieurs en agriculture, ESIA, 1993. -42 p.

158. Economies d'Asie et d'Amérique latine : changements de cap / Gilbert Etienne et Jean Revel-Mouroz, éd. - Genève : Ed. Olizane, 1993. - 196 p.

159.Education and science for maintaining biodiversity : programme and reports on preliminary study trips in Hungary, Côte d'Ivoire and Venezuela / an International Symposium on the occasion of the 172nd Annual meeting of the swiss Academy of Sciences, Basle, September 30th, and October 1 st and 2nd, 1992 ; [organized by the] Swiss National Commission for Unesco, Section Sciences and World Decade for Cultural Development. - Berne : Swiss National Commission for Unesco, [1992]. -112 p.

160. EGLI, Dominik. - Können Länderkredite profitabel sein ?. - Basel : Universität Basel, Institut für Volkswirtschaft, 1992. $-8 \mathrm{p}$.

161.Erosion, conservation, and small-scale farming / ed. by Hans Hurni and Kebede Tato. - Bern [etc.] : Geographica Bernensia [etc.], cop. 1992. - XIII, 582 p.

162. FAINI, Riccardo; MELó, Jaime de. - Adjustment, investment and the real exchange rate in developing countries. - Genève : Université de Genève, Faculté des sciences économiques et sociales, 1990. - 44, VI f. - (Cahiers du Département d'économie politique ; 90.08).

163. FAYE, Djidiack. - Désengagement étatique et stratégies de création d'emplois non salariés au Sénégal: L'Opération maîtrisards“ dans la boulangerie, la pêche et le transport. - Genève : Institut universitaire d'études du développement, 1992. -187 p. + annexes. - (Mémoire de recherche; no. 77).

164.FERRONI, Marco "Strukturanpassung und Armutbekämpfung in Entwicklungsländern", in : Aussenwirtschaft vol. 47, no. 3, 1992, p. 389-411.

31 165. FLOTTAU, Heiko. - Die Bande der Clans : die arabische Welt besser verstehen. Basel [etc.] : Herder, cop. 1992. - 185 p. - (Herder Spektrum ; Bd. 4126). 

habitacional alternativo en Mexico : el caso FONHAPO [Fondo Nacional de Habitaciones Populares] / prólogo de Jean-Claude Bolay. - Lausanne: Instituto de investigación sobre el entorno construido (IREC) ; E.P.F.L., 1993. - 33 p.

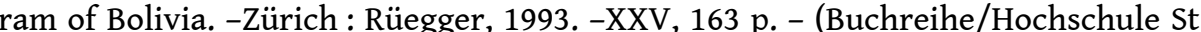
Gallen für Wirtschafts-, Rechts- und Sozialwissenschaft, Bereich Lateinamerikaforschung; Bd. 34).

41 175.HEINE, Ina; HEINE, Peter. - O ihr Musliminnen..: Frauen in islamischen Gesellschaften. - Basel : Herder, 1993. - 218 p. - (Spektrum ; Bd. 4217).

176.HOTTINGER, Arnold. - Islamischer Fundamentalismus. - Zürich: Neue Zürcher Zeitung, cop. 1993. - 207 p. Afrique du Sud rurale. - Genève : Université de Genève, Faculté de médecine, 1993. -65 f. 178. HUMBEL, Rainer. - Tea area changes in Sri Lanka: analysis of regional distribution, processes, mechanisms, and correlating factors of changes in the area cultivated with tea since 1956. - Zürich : University, Department of Geography, 1991. XI, 289 p. - (Sri Lanka studies ; [4]).

166. FONTANA, Jérôme. - Explosion démographique mondiale : maîtrise ou dérive ?: étude de croissance de population dans les pays du tiers monde, en particulier au Rwanda et en Chine. - Vésenaz, Genève : Fondation Avenir 2000,1992. - 95 p.

Chili : 1973-1993 / Jac Forton. Suivi de Paroles pour oublier l'oubli / d'Eduardo Galeano. - Genève : Centre Europe-Tiers Monde, 1993. - 248 p. + annexes. programmes in developing countries / under the dir. of Christian Morrisson. -Paris : Organisation for economic co-operation and development, 1992. - 80 p. -(Technical papers/OECD Development Centre ; № 59).

flushing : a case study in Namwawala, Kilombero Dostrict, Tanzania. - Zürich : Verlag der Fachvereine an den schweizerischen Hochschulen und Techniken, cop. 1993. -XX, 210 p., Thèse ETHZ 1992.

Huber, 1992.-341 p.

172. GOYENS, Philippe; LAMOTTE, Paul-Jacques. - La pédiatrie en Afrique : [guide pratique]. - Vevey : Nestec, cop. 1990. - 93 p., La couv. porte : Nestle Nutrition Services.

in Mexican manufacturing: 1980-1990. - Genève : Université de Genève, Faculté des sciences économiques et sociales, [ 1993]. - 29 p. - (Cahiers du Département d'économie politique. Université de Genève ; $n^{\circ}$ 93.08).

174. GRUETTER, Jürg. - A socio-economic evaluation of the structural adjustment 177. HUMAIR, Jean-Paul. - Suivi médical et compliance de patients hypertendus en

179. HURNI, Hans; PERICH, Isabel. - Towards a Tigray regional environmental and economic stragegy (TREES): a contribution to the Symposium on Combatting environmental degradation in Tigray, Ethiopia. - Berne : University of Berne, Institute 
of Geography, Group for development \& environment, 1992. - VIII, 32 p. -(Development and environment reports ; No. 6). actores públicos y privados en Bolivia: estudio sobre el papel del gobierno local. Vissoie ; Genève : Association suisse pour le service aux régions et communes (SEREC) ; I.U.E.D., 1993.-34 p. (bed development strategy: use and misuse of historical experiences. - Geneva University of Geneva, Centre of Insternational Economic History, cop. 1993. -110 p. (Monograph; no. 6). 
Zentrum für Zukunftsforschung, 1984. - 56 f. - (Dokumente/St. Galler Zentrum für Zukunftsforschung ; Nr. 7).

192. KRAUER, Alex. - Sustainable development: eine Chance für fortschrittliche Unternehmen : Referat anlässlich der Generalversammlung des Arbeitskreises Kapital und Wirtschaft vom 9. April 1992 in Zürich. - Zürich: Arbeitskreis Kapital und Wirtschaft, 1992. -15 p.- (Schriftenreihe Zeitthemen ; 8).

193. Landschaftsökologische Entwicklungsstrategien für Drittweltländer als methodisches Problem: Veröffentlichungen des 17. Basler Geomethodischen Colloquiums, [1992] = Ecological developing strategies for Third World-countries as a methodological problem: proceedings of the 17th Basle Geomethodological Colloquium, 1992 : Les stratégies du développement dans le domaine de l'écologie des paysages à destination du Tiers Monde en tant que problème méthodologique : le 17e Colloque méthodologique de Bâle, 1992. - Basel : Basler Afrika-Bibliographien, 1992. 165 p. - (Geomethodica ; vol. 17).

194. LEISINGER, Klaus M. - Hoffnung als Prinzip : Bevölkerungswachstum : Einblicke und Ausblicke. - Basel [etc.] : Birkhäuser, 1993. - 422 p.

61 195. LEYBOURNE, Marina. - Links between the steppe and cultivated areas through migration : the socio-economic organization of production of the semi-nomadic agropastoral society of the Syrian steppe. - Genève: Institut universitaire d'études du développement, 1993. - XII, 172 p., Diplôme de recherche ; no. 78.

196. LINIGER-GOUMAZ, Max. - Who's who de la dictature de Guinée équatoriale : les nguémistes, 1979-1993. - Genève : Les Ed. du Temps, 1993. - 351 p.

197. La littérature africaine francophone : 200 suggestions de lecture / avec une préf. de Pius Ngandu Nkashama. - Lausanne: Bibliothèque cantonale et universitaire; Déclaration de Berne, 1991. - 98 p.

198. LUCCHINI, Riccardo. - L'enfant de la rue et la consommation de drogue au Brésil : réflexions sur la toxicodépendance. - Fribourg: Université de Fribourg, Institut des sciences économiques et sociales, cop. 1992. - 48, XI p. - (Working papers ; no. 209).

199. M AN Y A, K'Omalowete a Djonga. - Evolution du pouvoir local au Sankuru : la légitimité et le rôle des chefs de groupements ainsi que leur place dans la formation des classes sociales au Zaïre. - Lausanne : Université de Lausanne, 1992. - 815 p., Thèse sc. pol.

200. MARIDOR, Raymond. - Réforme fiscale et lutte contre la pauvreté dans le cadre des programmes d'ajustement structurel. - Bern : DEH, 1993.

201.MARSHALL, Adriana. - Circumventing labour protection: non-standard employment in Argentina and Peru / with contrib. by Francisco Verdera V. and Isabel Yépez del Castillo. - Geneva : International institute for labour studies, 1992. - 149 p. (Research studies / International institute for labour studies ; 88).

202. MELO, Jaime de ; ROLAND-HOLST, David. - Industrial organization and trade liberalization: evidence from Korea. - Genève: Université de Genève, Faculté des sciences économiques et sociales, 1991. - 34, IV f. - (Cahiers du Département d'économie politique ; 91.14).

203.MENDIS, Patrick. - Human environment and spatial relationships in agricultural production: the case study of Sri Lanka and other tea producing countries. - Bern 
[etc.] : P. Lang, 1992. - XXIV, 256 p. - (American university studies. Series 16, Economics ; vol. 8). Conférence du Comité interrégional de coordination des associations régionales de développement (ICCDA), 24-27 avril 1990 à l'Institut international de planification de l'éducation (IIPE) ; avec lacollab. de l'UNESCO et le soutien de NORAD et FINNIDA.

204. MERCIAI, Patrizio. - Les entreprises multinationales en droit international. Genève : Université de Genève, 1992. - 294 p., Thèse sc. pol.

205. MONNIER, Laurent. - Démocratie et factions en Afrique centrale. - [S.I.] : La Revue nouvelle, 1993. - [11 ] p. - (Extr. de La revue nouvelle, jan-fév. 1993, no. 1 -2, p. 122-131).

206. MÜLLER, Q. - Ethiopie: dossier à l'attention des représetnants d'œuvres d'entraide et des bureaux de consultation juridique / trad, de O. von Allmen. Lausanne : Office central suisse d'aide aux réfugiés (OSAR), 1992. - [112] p.

207. POLIAKOV, Leon ; DELACAMPAGNE, Christian ; GIRARD, Patrick. - Rassismus : über Fremdenfeinlichkeit und Rassenwahn. -Zürich [etc.] : Luchterhand, 1992.

208. POZO, Maria Esther et al. - Strassenkinder : Austauschtreffen der Mädchen aus Bolivien : ein Hintergrundbericht mit Original-Aussagen / [Übers. : Elke Kalkowsky]; [Fotos : Diether Grünenfelder]. - Zürich : EcoSolidar, 1993. - $34 \mathrm{p}$.

209.REGNIER, Philippe. - The dynamics of small and medium-sized enterprises in Korea and other Asian NIEs (newly industrialized economies). -[S.I.] : Kluwer, 1993. [13] p., Extr. de Small Business Economics, NO. 5, 1993, p. 23-35.

210.REGNIER, Philippe ; NIU, Yuanming; ZHANG, Ruijun. -Toward a regional "block" in East Asia: implications for Europe. - Taipei, Taiwan: Institute of international relations, 1993. - [19] p., Extr. de : Issues and studies : [..], vol. 29, no. 3.

211. Le rôle de l'Etat dans les processus de développement : rapport final / Quatrième - Genève: Association européenne des instituts de recherche et de formation en matière de développement, 1990. $-49 \mathrm{p}$.

212. The Role of the state in development process / ed. by Claude Auroi; pubi, in collab. with the European Association of Development Research and Training Institutes (EADI). - London : Cass, 1992. - 263 p. - (EADI book series ; 15).

213. SABELLI, Fabrizio. - Recherche anthropologique et développement : éléments pour une méthode. - Neuchâtel ; Paris : Ed. de l'Institut d'ethnologie ; Ed. de la Maison des sciences de l'homme, 1993. - 176 p. - (Recherches et travaux ; 11).

1 214. SAlAZAR J., Alonso. - Des enfants tueurs à gages: les bandes d'adolescents de Medellin / trad, de l'esp. (Colombie) par Julian Garavito. - Genève : Centre Europe-Tiers Monde ; Ramsey, 1992. - 221 p. - (Documents et essais).

215. SAVIO, Roberto et al. - Où va l'Amérique latine ?: débat euro-américain autour du 500e anniversaire de la rencontre de deux mondes / [photographies de Jean Möhr].

- Genève : Institut universitaire d'études du développement, 1992. - 53 p.

216.SCHIESSER-GACHNANG, Doris. - Bolivien: Hyperinflation, Stabilisierung und Strukturanpassung : eine makroökonomische Analyse. - St. Gallen : Universität St. Gallen, 1993. - XVI, 343 p., Thèse sc. écon. 
VALDEZ CARRIZO, Edgar. - Autopsie de la maladie: soins et parcours thérapeutiques dans le système de santé à Vallegrande, Bolivie. - Genève : Institut universitaire d'études du développement, 1992. - VII, 231 p. - (Mémoire de diplôme de recherche; no. 70).

92 223. Vers un développement rural endogène en Afrique occidentale : actes du Colloque de Neuchâtel /organisé par l'Institut de géographie de l'Université de Neuchâtel les 7 et 8 juin 1991 ; Frédéric Chiffelle, réd. - Neuchâtel : Société neuchâteloise de géographie, 1992. - 103 p. - (Bulletin de la Société neuchâteloise de géographie ; no. 36).

93 224. "Vietnam", in : Vivant Univers No. 401, sept.-oct. 1992. - Fribourg : Missionnaires d'Afrique-Peres Blancs, 1992. - 48 p.

225. WARD, Haskell G. - The social dimension in the development of Africa. - Berne : Swiss Development Cooperation, 1993. - 11 p.

226. WIDJOJO, Ida Juda. - Erfolgskriterien und Erfolgskontrolling bei Inflation in einem Entwicklungsland am Beispiel Indonesiens. - Bern : Universität Bern, 1992. - X, 199 p., Those sc. pol.

227. ZNOJ, Heinzpeter. -Tausch und Geld in Zentralsumatra : zur Substantiven Theorie der Marktintegration. - Bern : Universität Bern, 1992. - IV, 317 p., [12] f., These phil.hist. 